\title{
Ureteral Injury During Difficult Gynaecological Surgeries: Report Of 2 Cases, Literature Review And The Way Forward.
}

\author{
${ }^{1}$ Usman Mohammed Tela, ${ }^{2}$ Adamu Sadiq Abubakar, ${ }^{3}$ Babagana Bako, \\ ${ }^{4}$ Anna Peter, ${ }^{5}$ Ado Dan'azumi Geidam, ${ }^{6}$ Abdu Mohammed Lawan \\ ${ }^{I}$ Department of Surgery, University of Maiduguri Teaching Hospital, Borno state, Nigeria. \\ ${ }^{2}$ Department of Anaesthesia and Intensive care, University of Maiduguri Teaching Hospital, Borno state, \\ Nigeria. \\ ${ }^{3}$ Department of Obstetrics and Gyaenecology, University of Maiduguri Teaching Hospital, Borno state, Nigeria. \\ ${ }^{4}$ Department of Obstetrics and Gyaenecology, University of Maiduguri Teaching Hospital, Borno state, Nigeria. \\ ${ }^{5}$ Department of Obstetrics and Gyaenecology, University of Maiduguri Teaching Hospital, Borno state, Nigeria. \\ ${ }^{6}$ Department of Surgery, University of Maiduguri Teaching Hospital, Borno state, Nigeria.
}

\begin{abstract}
Although iatrogenic ureteral injuries are uncommon, but continues to poses a great challenge of potencial injuries to the gyanaecologists and young surgeons, especially during difficult abdominal and pelvic surgeries. We present two cases of ureteral injuries in our centre which occurred during challenging gynaecolgical procedures. Extensive abdomino-pelvic adhesions and large pelvic tumours were the main risk factors in the two index cases. Sites of injuries and the risk factors were discussed. Thorough preoperative evaluation in anticipation of iatrogenic injuries, sound knowledge of surgical anatomy with experience, ureteral stenting before the main procedure, meticulous dissections and discouraging "blind clamping" during bloody procedures among others can prevent such injuries. High index of suspicion is important for early detection of the injuries.
\end{abstract}

Keywords: gynaecological, iatrogenic, ureter, injuries,

\section{Introduction}

Generally, trauma to ureters is quiet uncommon, especially from external violence (either from blunt or penetrating abdominal injuries ) than from iatrogenic injuries. The proximity of the female reproductive organs to the lower urinary tract poses the risk of urological injury during gynaecological surgery. Ureteral injuries from gynaecological operations is a well recognized serious complication with an incidence of $10-30 \%$ particularly during radical hysterectomies. ${ }^{1,2,3}$ Patients with malignancies and those with past history of multiple laparatomies resulting in adhesions tend to have high risk of ureteral injuries. While some injuries are evident intra-operatively, most (up to 70\%) are not immediately apparent and present later in the postoperative period. ${ }^{2}$ Such injuries are potential source of serious morbidities like renal failure and uretero-vaginal fistula among others, especially when the diagnosis and management are delayed. We present two cases of iatrogenic ureteral injuries which were immediately intervened. The literature review and the way forward were also discussed.

\section{Case 1}

AI was a 55 year old $\mathrm{P}_{2}{ }^{+1}, 1$ alive and her last child birth was 32 years before this index presentation. She was evaluated and prepared by the gynaecologists' for her $5^{\text {th }}$ laparatomy when she presented with a recurrent dull aching and localized suprapubic pain of a year duration. Associated slowly progressive suprapubic swelling, but no per-vaginal bleeding or abnormal vaginal discharge. There were no urinary tract or gastrointestinal symptoms and no associated weight loss. She had 4 different laparatomies in the last 23years, the first two were myomectomies on account of sub-fertility, later had total abdominal hysterectomy(TAH) and the last surgery was for right ovarian cystectomy for a symptomatic ovarian cyst. She was not a known diabetic or hypertensive patient.

Examination revealed an obese woman with Body Mass Index of $33 \mathrm{~kg} / \mathrm{M}^{2}$, not ill looking, not pale and no peripheral lymphadenopathy. Vital signs were within normal limit. Abdomen was full with midline surgical scar extending over a suprapubic swelling, about 20 weeks height, more toward the right side, not tendered with smooth surfaces and fixed to the underlying structures. The liver, kidneys and spleen were not palpable and no ascities. Vaginal, rectal examination and other systems were normal. An assessment of ovarian tumour was made. Abdominal ultrasound scan revealed huge multi-cystic mass with septations and thicken end wall containing fluid of homogenous echo patterns, other abdomino-pelvic organs were normal (as shown in fig 1). Renal function and full blood count were within normal limits and intra-venous urography(IVU) not done due to financial constraint. She was planned for bilateral ooperectomy. Operative findings were extensive adhesions 
involving the bowel and the highly vascular huge right ovarian mass. Mobilization and ooperectomy was done, the tumour was $20 \mathrm{~cm} \times 18 \mathrm{~cm}$, weighing $1.4 \mathrm{~kg}$, there was an iatrogenic transaction injury to the left ureter, shortly below the level where it crosses the iliac vessels. At that level, both the ureter and ovarian vessels were buried in adhesions, hence it was inadvertently involved while clamping and dividing the ovarian vessels. Urine was jetting at the transected point of the ureter. Multiple perforations of the jejunum and sigmoid colon also occurred. Urologist and general surgeon were invited intra-operatively, spatulated tensionless end to end anatomosis of the ureter over double $\mathrm{J}$ stent was done. Bowel perforations were repaired and defunctioning transverse colostomy done. Postoperative condition was satisfactory and histology of the tumour was mucinous cyst adenoma(benign). Colostomy was closed 4 weeks postoperatively and double J-stent was removed by cystoscopy after 6 weeks postoperative period.

\section{Case 2}

YLA was a 42 year old $\mathrm{P}_{1}{ }^{+0} 1$ alive, her last child birth was 19 years prior to index presentation and menstrual period was 3 weeks prior to presentation. She presented to the Gynaecologists with irregular menstrual period of 3 months duration. She started having prolonged menstrual period and menorrhagia with frequent changes of sanitary pads. There were associated dizziness, palpitation and easy fatiguability. She noticed painless suprapubic swelling which was progressively increasing in size. She was transfused with 3 pints of whole blood for the same complain on the account of anaemia earlier. It was not associated with weight loss and no significant gastrointestinal or urinary symptoms. She attend menarche at the age of 17 , menstruates for 6 days in a regular cycle of 28-30days. She has been on anti-hypertensive and anti-epileptic drugs for 13 years, but not a known diabetic.

On examination she was ill looking in depressed mood, pale, aniecteric, not dehydrated and no pitting pedal oedema. Pulse rate was $120 / \mathrm{min}$ with blood pressure of $80 / 60 \mathrm{mmHg}$, heart sounds were 1and 2 only, no murmurs. There was suprapubic mass arising from the pelvis with 34weeks height, mobile with nodular surface and other abdominal organs were normal. Vaginal examination revealed uterine mass with closed cervical os. Other systems were normal. An assessment of uterine fibroid with anaemia was made. The admitting packed cell volume was $18 \%$, abdominal ultrasound scan revealed multiple uterine fibroids with serosal and intramural locations and the largest was $8.2 \mathrm{~cm}$ in diameter while other organs were normal. Renal function test was also normal She was transfused whole blood and optimized, then scheduled for Total Abdominal Hysterectomy. Operative findings were huge nodular uterus surrounded by extensive pelvic adhesions, the hysterectomy specimen was $29 \mathrm{~cm}$ by $17 \mathrm{~cm}$ in size and weighed $4.1 \mathrm{~kg}$. There was iatrogenic avulsion injury to the left ureter at uretero- vesical junction alerted by accumulation of urine in the pelvis, for which urologist was invited and a non-refluxing tensionless uretero-neocystostomy was done over a doube J-stent (as shown in figure 2). Postoperative condition was satisfactory. She was seen during follow up (6 weeks postoperatively) and the stent was removed by cystoscopy.

\section{Discussion}

Sound knowledge of surgical anatomy is of paramount importance during surgery in the pevic region and retro-peritoneal space. The pair of ureters are cord-like non pulsating hollow organs, whose wall includes 3 muscle layers responsible for its peristaltic wave of movement, responsible for transporting urine from the pelvis of the kidneys to the bladder. Each ureter is about 25 to $30 \mathrm{~cm}$ in length and $3 \mathrm{~mm}$ in diameter They run down in retroperitoneal space behind the ascending and descending colons, medial to the greater psoas muscle. On reaching the pelvic cavity, each crosses the bifurcation of the common iliac vessels on both side, then crosses the ovarian vessels (first critical point of injury). This corresponds with the point of injury in the index case 1 presented. The ureters form the posterior border of the ovarian fossa; they progress distally to the lateral edge of the uterosacral ligaments (second critical point of injury) up to the point of the cardinal ligament. At the base of the cardinal ligament, the ureter passes under the uterine artery (third critical point of injury), at the level of the ischial spine. At this point it goes initially forward and then medially, under the wide extensive ligament, between the uterine and vaginal arteries, toward the bottom of the lateral sac of the vagina where it then passes approximately $2 \mathrm{~cm}$ lateral to the cervix. Here, the ureter ascends anterior to the vagina for a short distance finally reaching the base of the bladder, where it opens at a lateral angle of the vesical trigone, $5 \mathrm{~cm}$ away obliquely perforating the wall of the bladder (this is fourth critical point of injury) ${ }^{4}$, which corresponds with the injuries that occurred in the index case 2 presented, due to extensive adhesion in the region that distorted the visualization of the ureter, and hence inadvertently transacted during sharp dissection.. The upper part of the ureter gets it blood supply from multiple sources coming from the medial side of the ureter, while the lower part is supplied by branches of the arteries coming from the lateral side, ${ }^{5}$ as shown in figure 3 . Anatomic variation of the course of the ureters probably contribute to injuries in about $0.5 \%$ of cases. ${ }^{6}$

Risk factors for iatrogenic ureteral injury comprises of techinical, anatomical and anatamopathological factors. ${ }^{7}$ Technical difficulties in controlling massive intraoperative bleeding during pelvic tumour 
surgeries may force the gynaecologist to blind clamp and even suture the bleeding point and the near-by ureter may be inadvatently clamped or even ligated together. Operation on huge pelvic tumour may be technically difficult for the inexperienced surgeon to mobilize it, hence the ureters may be injured as it happened in the $2^{\text {nd }}$ index case presented. Anatomically, the ureter is located in the retroperitoneal space, occasionally it can be mistaken for a fold of peritoneum and therefore be transected during sharp dissection. Its precarious blood supply can easily be damaged during excessive mobilization and skeletanization and hence results in avascular necrosis of its segment. Pathological changes in the pelvis because of adhesions from previous surgeries as in the index cases 1 and 2, endometriosis, radiotherapy and others can subject the ureter to injury during dissection. Previous study also reported on pelvic neoplasia and adhesions as risk factors, similar to the two cases we reported $^{8}$. Also lack of background knowledge of the existing congenital anomalies of the ureters in the patient before surgery, may lead to ureteral injury. Presence of urinoma and or empty urinary bladder intraoperative may suggest ureteral injury. Occasionally there may be need to do on table intravenous pyelography or injection of $1-2 \mathrm{ml}$ of methyline blue into the renal pelvis can ease at locating the site of injury.

Iatrogenic injuries are best managed immediately at the time of injuries under general or regional anaesthesia. Hence, efforts should be made right in the intraoperative period ${ }^{10}$. Mild injuries from clamping the ureter or ligature discovered at any site during the course of surgery should be removed immediately and the ureter inspected. If there is any suspicion of ischemia or damage to the ureteral wall, a ureteral stent should be placed by cystoscopy or ureterotomy and removed after 6 weeks ${ }^{11}$. If the ligature has caused extensive damage to the ureter, then it is preferable to excise the damaged segment and re-implant to the bladder or re-anastamose the two ends of the ureter over a stent. Severe ureteral injuries such as lacerations or transections below the pelvic brim (lower third) is also managed by re-implantation wih psoas hitch or Boari flap when lost segment is greater tha $4 \mathrm{~cm}$ and when the injuries are above the pelvic brim (middle and upper third) it can be managed as shown in figure 4. Uretero-ileal interposition can be an alternative to transuretero-ureterostomy, when there is significant lost of length. The principles of the ureteral anastamosis comprises of spatulated and water- tight tensionless anastamosis.

General preventive measures include good grasp of surgical anatomy, thorough patient evaluation in an anticipation for potential ureteral injury in the pelvic surgical procedure to be done. The inexperienced surgeon should be supervised. Cystoscopic ureteral stenting or open bladder cannulation of ureters can be done before the main pelvic surgery ${ }^{12}$. During abdominal hysterectomy, the intracapsular technique of ligation and sectioning of the cardinal, uterosacrate and vesico-uterine ligaments should be as close as possible to the uterus. Also ligation of the uterine vessels should be close to the uterus, this is the most frequent point of ureteral injury in the sub-ligamentar segment of the pelvine area. Ligation and sectioning of the lumbo-ovarian ligament should be close to the ovary. During vaginal hysterectomy, proper exposure of vesico- uterine area will safeguard the ureter in pre-ligamentar segment of the pelvine area. Dissection in lateral should be discouraged during anterior colporaphy. During laparoscopy, coagulation of fallopian tube should be done with clear vision and after its isolation because the ureter is close to it ${ }^{7}$

\section{Figures}

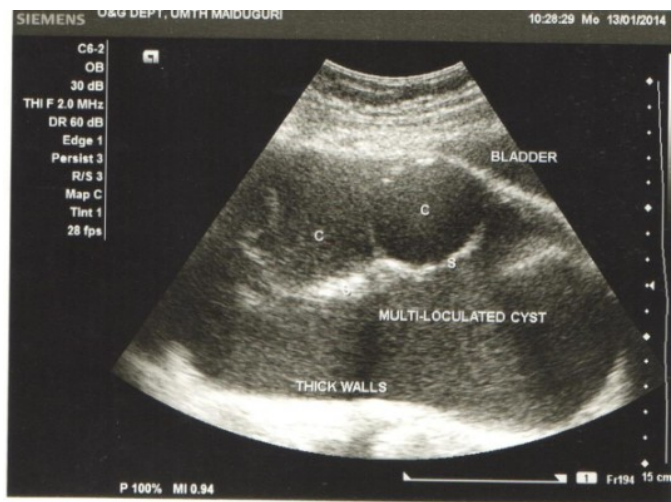

Figure 1a: Multicystic mass of case 1

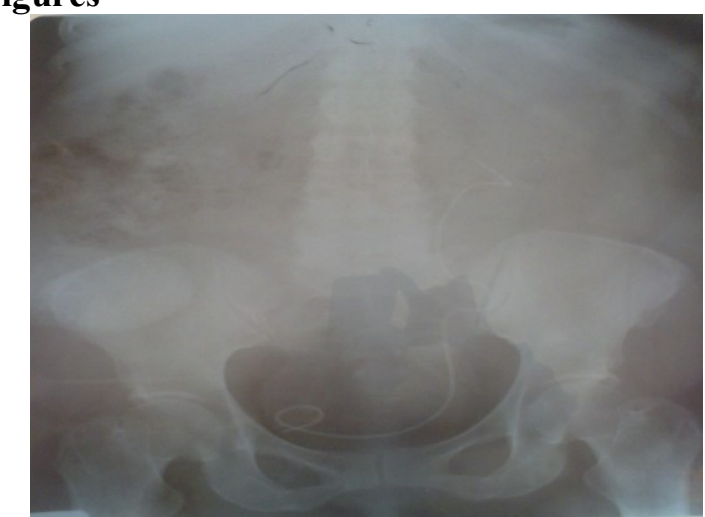

Figure 1b: Intact left ureteral stent of case2 


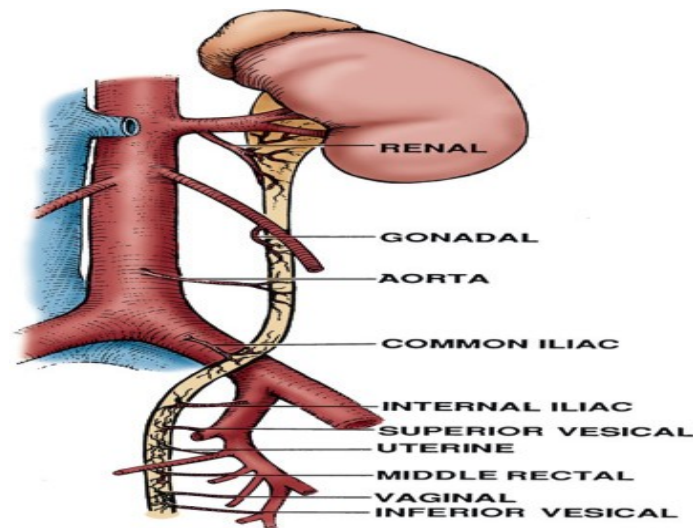

Figure 1c: Blood supply to the ureter ${ }^{5}$

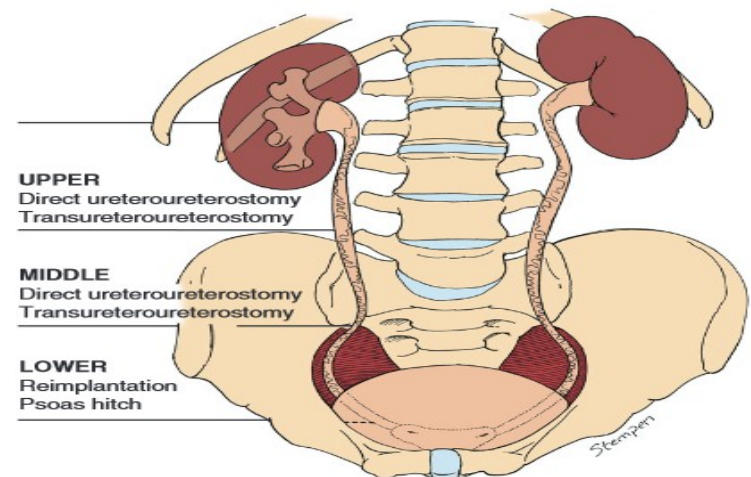

Figure 1d: Management options for ureteral injuries ${ }^{8}$

\section{Conclusion}

Iatrogenic ureteral injuries is preventable when anticipated and well prepared towards it in challenging surgeries. Presence of pelvic adhesions and large pelvic tumors were the risk factors in the cases presented. Such injuries can be prevented. Where it occurs, high index of suspicion is crucial for early detection of cases. Immediate intra-operative interventions remains the best form of management.

\section{References}

[1]. Vito Leanza, Antonia Francesca Di Prima, Gianluca Leanza, Maria Cristina Teodoro, Antonio Carbonaro, et al. How to Prevent Ureteral Injuries during Pelvic Gynaecological Procedures. Journal of Applied Medical Sciences, 2013; 2(3): 2241-2328.

[2]. Carlton CE, Scott R, Guthrie AG: The initial management of ureteral injuries: a report of 78 cases. J Urol 1971 Mar; $105(3)$ : $335-$ 40.

[3]. Davis, D M: Intubated ureterotomy: a new operation for ureteral and ureteropelvic strictures . Surg Gynecol Obstet 1943; 76: 851866.

[4]. Manoel Afonso Guimarães Gonçalves, Fernando Anschau, Daniela Martins Gonçalves and Chrystiane da Silva Marc: "Ureter: How to Avoid Injuries in Various Hysterectomy Techniques" Text book of Hysterectomy, chapter 18, page 286

[5]. James Kyle Anderson, Jeffrey A. Cadeddu: Surgical Anatomy of the Retroperitoneum, Adrenals,Kidneys and Ureters; CampbellWalsh Urology, $10^{\text {th }}$ Edition, Vol.1, chapter1,p.31

[6]. Mathew P., Valencia P., Cousin C., et al. Operative injuries during vaginal hysterectomy. Eur. J. Obstet. Gynaecol. Reprod. Biol. 2001; 97: 71-75

[7]. Cirstoiu M, Munteanu O: Strategies of preventing ureteral iatrogenic injuries in obstetrics-gynecology, Journal of Medicine and Life. 2012; 5(3):277-279.

[8]. Liapis A, Bakas P, Giannopoulos V, Creatsas G. Ureteral injuries during gynecological surgery. Int Urogynecol J Pelvic Floor Dysfunct 2001; 12(6): 391-3

[9]. Santucci RA, Doumanian LR. Upper Urinary Tract Trauma. Campbell-Walsh Urology, $10^{\text {th }}$ Edition, Vol 2, chapter 42, p.1183

[10]. Redan JA, McCarus SD. Protect the ureters. JSLS 2009; 13:139.

[11]. Gill H, Broderick GA: Urologic complications of gynecologic surgery. Vol. 13. AUA Update Series; 1994: 254-9.

[12]. Spence HM, Boone T: Surgical injuries to the ureter . JAMA. 1961; 176(13): 1070-1076. 